\title{
Solid-solid phase transition in hard ellipsoids
}

\author{
M. Radu, ${ }^{1}$ P. Pfleiderer, ${ }^{2}$ and T. Schilling ${ }^{1, a)}$ \\ ${ }_{1}^{1}$ Institut für Physik, Johannes Gutenberg-Universität, Staudinger Weg 7, D-55099 Mainz, Germany \\ ${ }^{2}$ Department of Chemical Engineering, K. U. Leuven, W. de Croylaan 46, B-3001 Leuven, Belgium
}

(Received 7 August 2009; accepted 28 September 2009; published online 30 October 2009)

\begin{abstract}
We present a computer simulation study of the crystalline phases of hard ellipsoids of revolution. A previous study [P. Pfleiderer and T. Schilling, Phys. Rev. E 75, 020402 (2007)]. showed that for aspect ratios $a / b \geq 3$ the previously suggested stretched-fcc phase [D. Frenkel and B. Mulder, Mol. Phys. 55, 1171 (1985)] is unstable with respect to a simple monoclinic phase with two ellipsoids of different orientations per unit cell (SM2). In order to study the stability of these crystalline phases at different aspect ratios and as a function of density we have calculated their free energies by thermodynamic integration. The integration path was sampled by an expanded ensemble method in which the weights were adjusted by the Wang-Landau algorithm. We show that for aspect ratios $a / b \geq 2.0$ the SM2 structure is more stable than the stretched-fcc structure for all densities above solid-nematic coexistence. Between $a / b=1.55$ and $a / b=2.0$ our calculations reveal a solid-solid phase transition. (C) 2009 American Institute of Physics. [doi:10.1063/1.3251054]
\end{abstract}

\section{INTRODUCTION}

Suspensions of hard particles (i.e., particles that interact via an infinitely strong, repulsive excluded-volume interaction potential) have been successfully used as model systems for the statistical mechanics of liquids and solids for more than half a century. For this class of system phase transitions are entropy rather than enthalpy driven, and the relevant control parameters are the particle shape and concentration rather than temperature. Hard ellipsoids are a simple model for systems whose macroscopic properties depend on the interplay of positional and orientational entropy such as liquid crystals $^{1-4}$ and orientational glasses ${ }^{5-7}$

In recent years it has been shown by computer simulations and experiments that randomly packed arrangements of hard ellipsoids can reach densities much higher than random close packing of spheres. ${ }^{8-10}$ At certain aspect ratios, random packing of ellipsoids can even reach densities almost as high as the closest crystalline packing of spheres. ${ }^{8}$ However, this does not imply that random packing of ellipsoids is as dense as their densest known crystalline packing. In 2004, Donev et $a{ }^{11}{ }^{11}$ introduced a family of crystalline packings of ellipsoids, which reach a packing fraction of $\eta \simeq 0.7707$ (as compared with $\eta=\pi / \sqrt{18} \simeq 0.7405$ for the fcc packing of spheres and stacking variants thereof).

Inspired by this study, we reexamined the phase diagram of hard ellipsoids of revolution. ${ }^{12}$ We found that the stretched-fcc phase, which had before been assumed to be the stable crystalline phase, ${ }^{13}$ was unstable with respect to a different crystalline phase. The more stable structure has a simple monoclinic unit cell containing two ellipsoids of unequal orientation (SM2) (cf. Fig. 1). The packings constructed by Donev et al. ${ }^{11}$ are a special case of SM2 (the infinite-pressure limit).

At that time we did, however, not compute free energy

${ }^{a)}$ Electronic mail: schillit@uni-mainz.de. differences between SM2 and stretched fcc. In the present article we report on Monte Carlo (MC) simulations in which SM2 and stretched fcc are connected to their respective harmonic crystals ["Einstein crystals" (ECs)] via thermodynamic integration (TI), and hence their free energies are determined. In order to sample the TI pathway, we adapted the Wang-Landau algorithm. ${ }^{14}$ In the original Wang-Landau scheme a flat histogram of the internal energy is constructed. Here we constructed a flat histogram of the coupling parameter that couples the hard ellipsoid model to the EC instead.

\section{METHOD OF COMPUTATION}

In order to determine which of the two phases is thermodynamically more stable, one compares their relevant thermodynamic potentials, e.g., in the case of constant par-

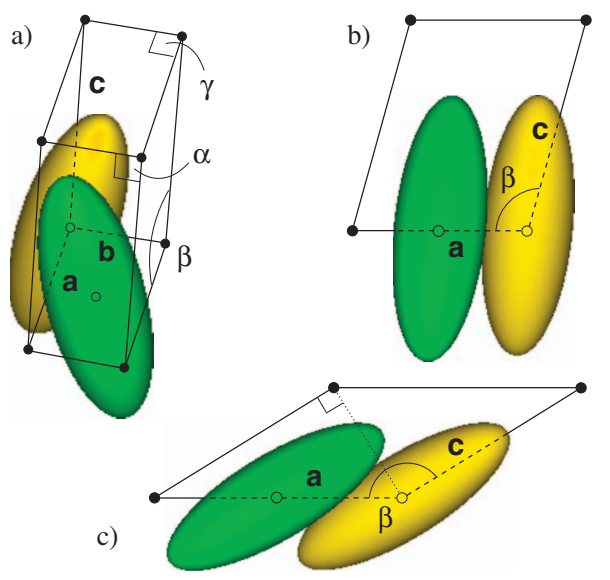

FIG. 1. Unit cell of SM2 (Ref. 12) with $a / b=3$. The open circles indicate the centers of the two ellipsoids that form the basis. The yellow (light gray) ellipsoid is at the origin, the green (dark gray) one is at $\frac{1}{2}(\mathbf{a}+\mathbf{b})$. The cell is monoclinic. $\beta$ is the soft degree of freedom. Part (c) shows the cell at close packing (the infinite-pressure limit), where it is an instance of the family of packings introduced by Donev et al. (Ref. 11). Note the indicated right angle and the resulting symmetry about the $b c$-plane in this case. 


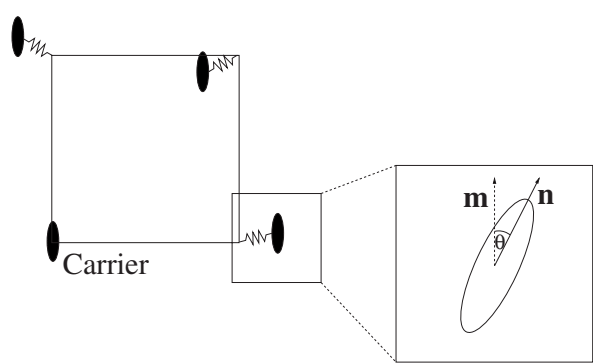

FIG. 2. Scheme of an EM for hard ellipsoids.

ticle number $N$, volume $V$, and temperature $T$, with their free energies $F$. Within a MC simulation, however, for most models it is impossible to compute $F$ because of its direct connection to the accessible phase space volume $\left(\boldsymbol{q}^{N}, \boldsymbol{p}^{N}\right)$. To solve this problem the method of $\mathrm{TI}^{15,16}$ is commonly used, in which the free energy difference between the system of interest and a reference system can be calculated by introducing an artificial external potential $U$, such that

$$
\Delta F=F_{\text {sys }}-F_{\text {ref }}=\int_{\zeta=1}^{\zeta=0} \mathrm{~d} \zeta\left\langle\frac{\partial U\left(\boldsymbol{q}^{N} ; \zeta\right)}{\partial \zeta}\right\rangle_{\zeta} .
$$

Here, the parameter $\zeta \in[0,1]$ links the interaction potential of the system of interest $U_{\text {sys }} \equiv U(\zeta=0)$ to the potential of the reference system $U_{\text {ref }} \equiv U(\zeta=1)$ by

$$
U\left(\boldsymbol{q}^{N} ; \zeta\right)=(1-\zeta) U_{\mathrm{sys}}\left(\boldsymbol{q}^{N}\right)+\zeta U_{\mathrm{ref}}\left(\boldsymbol{q}^{N}\right) .
$$

During a typical integration, $U_{\text {sys }}$ is gradually switched on and at the same time $U_{\text {ref }}$ is gradually switched off. However, the hard-core interaction of the ellipsoids does not allow for a gradual change. Therefore, $U_{\text {sys }}$ is imposed in the first step, and then $U_{\text {ref }}$ is gradually switched off in the second. With this procedure, the free energy of the system can be calculated as $F_{\text {sys }}=F_{\text {ref }}+\Delta F_{1}+\Delta F_{2}$, where the subscripts refer to the two steps just described,

$$
\Delta F_{1}=-\ln \left\langle\exp \left[-\beta U_{\text {sys }}\right]\right\rangle_{\zeta=1},
$$

where $U_{\text {sys }}\left(\boldsymbol{q}^{N}\right)$ here is the overlap potential of the ellipsoids, and the configuration $\boldsymbol{q}^{N}$ consists of positions and orientations $\boldsymbol{q}^{N} \equiv\left\{\boldsymbol{r}^{N}, \theta^{N}\right\} .\langle\cdots\rangle_{\zeta}$ refers to the ensemble average where the potential is parametrized by Eq. (2). $\Delta F_{2}$ will be discussed together with the free energy of the reference state in the following paragraph.

From Eq. (1) it is obvious that $F_{\text {ref }}$ needs to be known from other sources, e.g., by analytical computation, and that no phase transition may occur during the integration process. In order to construct such a reference system, we consider a system of hard ellipsoids in which all particles except for one are coupled to the sites of a lattice via harmonic springs. The remaining particle is fixed in space and is called the carrier of the lattice. We fix this particle to the origin of the coordinate system. As we are interested in anisotropic particles, we will also restrict their rotational motion by a contribution $U_{\text {rot }}\left(\theta_{i}^{N}\right)$ to the potential. We set this to be proportional to $\sin ^{2} \theta_{i}$, where $\theta_{i}$ is the angle between the axis of particle $\boldsymbol{n}_{i}$ and a reference axis $\boldsymbol{m}_{i}$ (cf. Fig. 2).

This kind of model is known as an Einstein molecule (EM). ${ }^{17}$ [The reason for fixing one particle is the following.
In the case of an EC, center of mass motion of the entire system does not cost energy. Hence, for weak coupling one needs to shift all particle positions after every move to keep the center of mass positioned, as it was done, e.g., in the work of Polson et al. ${ }^{18}$ In the case of the EM, the fixed carrier particle ensures nondivergency of the center of mass mean square displacement for a negligible harmonic potential (see also Ref. 17).]

The interaction potential of the EM is

$$
\begin{aligned}
U_{\text {ref }} & =\sum_{i}{ }^{\prime} \lambda_{i}^{\text {trans }}\left(\boldsymbol{r}_{i}-\boldsymbol{r}_{0, i}\right)^{2}+\sum_{i}{ }^{\prime} \lambda_{i}^{\mathrm{rot}} \sin ^{2} \theta_{i} \\
& =\lambda \sum_{i}{ }^{\prime}\left[\left(\boldsymbol{r}_{i}-\boldsymbol{r}_{0, i}\right)^{2}+\sin ^{2} \theta_{i}\right],
\end{aligned}
$$

where $\boldsymbol{r}_{0, i}$ denote the position vectors of the lattice sites. The prime denotes that the sum runs over all particles except for the carrier. For simplicity we chose the spring constants of all lattice sites in the second line of Eq. (4) as equal. In addition we set $\lambda^{\text {trans }}=\lambda^{\text {rot }} \equiv \lambda$. We use twice the short axis $b$ as the unit of length and $k_{B} T$ as the unit of energy (except where stated otherwise). With this the unit of $\lambda$ is $k_{B} T /(2 b)^{2}$. As we are only interested in the configurational part of phase space, the kinetic energies of the particles are disregarded.

In order to evaluate the configurational part of the partition function of the EM, we assume that the maximum coupling constant $\lambda_{\max }$ is strong enough for $\theta_{i} \ll 1$ (see Appendix). So we obtain (cf. Ref. 19)

$$
\begin{aligned}
\frac{F^{\mathrm{FCC}}}{N}= & \frac{1}{N} \ln \left[\frac{N}{4 \pi V}\right]+\frac{3}{2}\left(1-\frac{1}{N}\right) \ln \left[\frac{\lambda_{\max }}{\pi}\right] \\
& +\left(1-\frac{1}{N}\right) \ln \left[\frac{\lambda_{\max }}{2 \pi}\right] .
\end{aligned}
$$

In case of the SM2-EM the same approach leads to

$$
\begin{aligned}
\frac{F^{\mathrm{SM} 2}}{N}= & \frac{1}{N} \ln \left[\frac{N}{8 \pi V}\right]+\frac{3}{2}\left(1-\frac{1}{N}\right) \ln \left[\frac{\lambda_{\max }}{\pi}\right] \\
& +\left(1-\frac{1}{N}\right) \ln \left[\frac{\lambda_{\max }}{2 \pi}\right] .
\end{aligned}
$$

The derivation of Eq. (6) is outlined in the Appendix. The difference in free energy per particle between the fcc-EM and the SM2-EM is $(\ln 2) / N$ due to the presence of two types of lattice sites in the SM2 unit cell. This difference vanishes in the thermodynamic limit $N \rightarrow \infty$.

Coming back to the calculation of $F_{\text {sys }}$ we rewrite the integral in Eq. (1) (with $\zeta=\lambda / \lambda_{\max }$ ) as

$$
\Delta F_{2}=\int_{\lambda_{\max }}^{0} \mathrm{~d} \lambda\left[\left\langle\sum_{i}{ }^{\prime}\left(\boldsymbol{r}_{i}-\boldsymbol{r}_{0, i}\right)^{2}\right\rangle_{\lambda}+\left\langle\sum_{i}{ }^{\prime} \sin ^{2} \theta_{i}\right\rangle_{\lambda}\right] .
$$

In order to perform the TI, we make use of the following idea: For two adjacent values $\lambda_{i}$ and $\lambda_{j}$, the parts of configuration space that contributes dominantly to the thermodynamic average in Eq. (7) overlap considerably. There are many expanded ensemble and flat histogram techniques that employ overlapping histograms in order to compare free energies of a system at different values of a reaction coordinate 
TABLE I. Results for the free energy of hard spheres.

\begin{tabular}{cll}
\hline \hline$\varrho$ & $N$ & \multicolumn{1}{c}{$F / N$} \\
\hline \multirow{2}{*}{1.04086} & 504 & $4.924(12)$ \\
& 768 & $4.957(10)$ \\
& 810 & $4.962(10)$ \\
1.09975 & 1728 & $4.988(7)$ \\
1.15000 & 1728 & $5.647(7)$ \\
\hline \hline
\end{tabular}

(e.g., free energy differences between the liquid and the solid phase, between different conformations of a molecule, etc.). Here, we use the coupling constant $\lambda$ as a "reaction coordinate" and evaluate Eqs. (3) and (7) by the following expanded ensemble technique: We discretize the range of values for $\lambda$. Then, besides translational and rotational moves, we perform a move in which the system passes from one model with a value $\lambda_{i}$ into an adjacent one with $\lambda_{j}$ and vice versa. In order to ensure good statistics when sampling the $\lambda$-range, we introduce a set of weights $\psi_{m}$ and sample the expanded ensemble given by the partition function

$$
\mathcal{Z}=\sum_{m=1}^{M} \mathcal{Z}_{m}\left(\lambda_{m}\right) e^{\psi_{m}}
$$

where $\mathcal{Z}_{m}\left(\lambda_{m}\right)$ is the partition function of the model $m$ with $\lambda=\lambda_{m}$ and $\psi_{m}$ its weighting factor. The acceptance probability of a $\lambda$-move is then given by

$$
P_{i \rightarrow j}=\min \left[1, \frac{\exp \left(\psi_{j}-U_{j}\right)}{\exp \left(\psi_{i}-U_{i}\right)}\right],
$$

such that for an adequate set of weights the system can be forced to visit the states of interest. One can then compute the free energy difference as

$$
F_{j}-F_{i}=-\ln \left[\frac{p_{j}}{p_{i}}\right]+\psi_{j}-\psi_{i} .
$$

Here $p_{i}$ and $p_{j}$ are the probabilities for the system to visit model $i$ or model $j$, respectively, in the presence of the weights.

The success of this procedure depends on finding appropriate weights. The weights are not known a priori, but they can be adjusted iteratively during the simulation, as has been introduced by Wang and Landau ${ }^{14}$ for the case of the density of states as a function of energy. We apply this idea to TI. Initially, we choose the weighting factors as $\psi_{i}=0 \quad \forall i$ $\in\{0, \ldots, M\}$. Then simulations for each subensemble are carried out in which after each $\lambda$-move the weight of the rejected model is increased by $\Delta \psi=1$. This leads to an increase in both the possibility to visit the accepted model and the possibility to stay there. As in the original algorithm $\Delta \psi$ is decreased by a factor $a<1, \Delta \psi \rightarrow a \cdot \Delta \psi$ (here, $a=0.5$ ), as soon as the difference between the probabilities becomes sufficiently small. Once the simulations are finished $p_{m} \approx p_{m-1}$ (in fact $\ln \left[p_{m} / p_{m-1}\right]$ was less than $10^{-5} N k_{B} T$ after only $10^{5}$ steps).

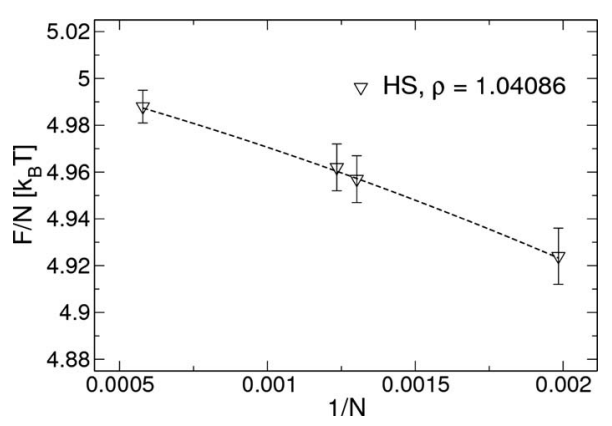

FIG. 3. Hard spheres: Free energy per particle as a function of the inverse particle number. Symbols: MC data. Line: Fit according to Eq. (11).

Finally, we consider the computation of $\Delta F_{1}=F_{\text {off }}-F_{\text {on }}$, where the $\lambda$-step does not change $\lambda$ but consists of switching on and off the hard-core potential.

According to Eq. (9) moves that switch off the potential or that lead to a state with no overlap are always accepted, whereas moves of the form off $\rightarrow$ on, which yield a state with at least two overlapping particles, are always rejected. For this case the coupling parameter was $\lambda=\lambda_{\max }$ (i.e., the reference state), and hence the free energy difference between the states on and off was expected to be very small.

Therefore we set the corresponding weights equal to 0 and kept them fixed during the calculation. (This approach is validated by our results for $\Delta F_{1}$, which were of the order of $10^{-4} N k_{B} T$.)

\section{RESULTS}

\section{A. Hard spheres}

In order to test the algorithm before applying it to anisotropic particles, we first computed the free energy of hard spheres at various densities $\varrho=N / V$ and particle numbers $N$. Table I summarizes our results. Figure 3 shows the free energy per particle as a function of $1 / N$ for $\varrho=1.04086$. The dotted line is a fit to

$$
\frac{F}{N}=e_{1}+\frac{e_{2}}{N}+\frac{e_{3}}{N^{2}}
$$

by which we extrapolate our results to infinite $N, F / N$ $=5.008(4) k T$ (see also Ref. 17).

Figure 4 shows a comparison of our results to EC and

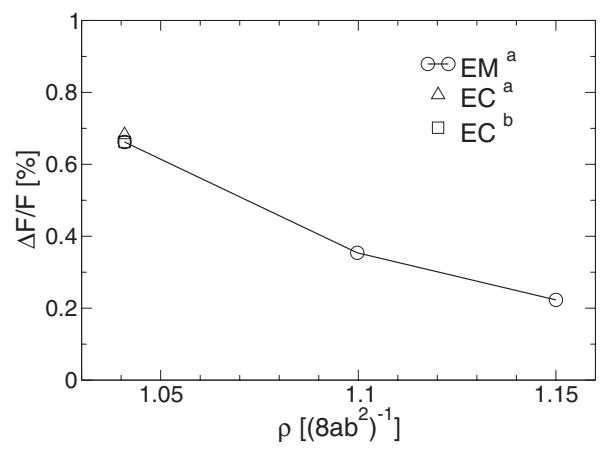

FIG. 4. Hard spheres: Relative difference $\Delta F$ (in percent) between our results and free energies computed by similar methods [(a) Ref. 17 and (b) Ref. 18]. There is a very good agreement. 


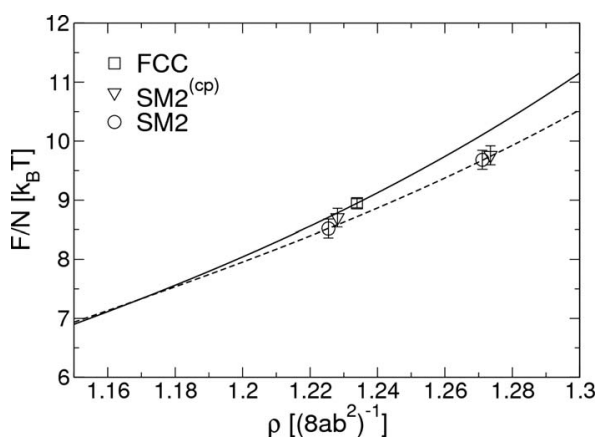

FIG. 5. Hard ellipsoids, $a / b=3$ : Free energy per particle as a function of density. Symbols are direct MC results for the free energy, lines are fits to $\mathrm{MC}$ data for the equation of state.

EM computations that did not use the Wang-Landau algorithm. There is a very good agreement, giving us confidence in the results for the hard ellipsoid case.

\section{B. Hard ellipsoids}

Previous work showed that the angle of inclination of the SM2 unit cell $\beta$ is a very "soft" degree of freedom, ${ }^{12}$ i.e., the corresponding shear modulus is almost zero. $\beta$ fluctuated strongly even at a pressure as high as $P=46 k_{B} T / 8 a b^{2}$ [for $a / b=3$, where the nematic-solid coexistence pressure is $P$ $=31 k_{B} T / 8 a b^{2}$ (Ref. 1)]. This unusual mechanical property is due to the fact that planes of equally oriented particles can slide across each other without much interaction, unless the system is forced to pack very densely. In order to quantify this effect, we computed free energies for various fixed values of $\beta$. In the special case that the unit cell is invariant under reflections with respect to the $b c$-plane [see Fig. 1(c)], the configuration has the same symmetry as (but different unit cell parameters than) the close-packed structure constructed by Donev et al. ${ }^{11}$ In the following we refer to this structure as $\mathrm{SM} 2^{(\mathrm{cp})}$.

Figure 5 shows the free energy for $a / b=3$ as a function of the density $\varrho$ (to simplify the comparison to other studies we use $1 / 8 a b^{2}$ as the unit of density here instead of $1 / 8 b^{3}$ ). The symbols are direct simulation results of free energies: Triangles for SM2 ${ }^{(\mathrm{cp})}$, circles for SM2 with different values of $\beta$ (see Table II for details), and a square for stretched fcc. The solid lines are polynomial fits to the equation of state data from our previous work (Ref. 12), integrated over $\varrho$ and shifted by a constant to fit the free energy data.

Taking the errors into account, there is no evidence for a difference in free energy between the different angles of in-

TABLE II. Hard ellipsoids: Free energy per particle for $a / b=3$.

\begin{tabular}{|c|c|c|c|c|}
\hline Lattice type & $\varrho$ & $N$ & $\begin{array}{c}\beta \\
(\operatorname{deg})\end{array}$ & $F_{\text {sim }} / N$ \\
\hline $\mathrm{fcc}$ & 1.23390 & 432 & (90) & $8.95(8)$ \\
\hline \multirow[t]{2}{*}{ SM2 } & 1.22545 & 432 & 110.76 & $8.52(16)$ \\
\hline & 1.27111 & 432 & 115.91 & $9.68(16)$ \\
\hline \multirow[t]{2}{*}{$\mathrm{SM} 2^{(\mathrm{cp})}$} & 1.22815 & 432 & 148.35 & $8.71(16)$ \\
\hline & 1.27352 & 432 & 147.97 & $9.76(16)$ \\
\hline
\end{tabular}

TABLE III. Hard ellipsoids: Free energy and Gibbs free energy per particle $\left(P=46 k_{B} T / 8 a b^{2}\right)$.

\begin{tabular}{lcccccc}
\hline \hline & & \multicolumn{5}{c}{$\beta$} \\
Lattice type & $a / b$ & $\varrho$ & $N$ & $(\mathrm{deg})$ & $F / N$ & $G / N$ \\
\hline fcc & 1.55 & 1.23027 & 1728 & $(90)$ & $7.18(7)$ & $44.57(12)$ \\
& 2.00 & 1.23171 & 1728 & $(90)$ & $8.24(7)$ & $45.58(12)$ \\
& 3.00 & 1.23390 & 432 & $(90)$ & $8.95(8)$ & $46.23(13)$ \\
SM2 & 2.00 & 1.27215 & 432 & 142.44 & $8.96(23)$ & $45.12(25)$ \\
& 3.00 & 1.27111 & 432 & 115.91 & $9.68(16)$ & $45.87(19)$ \\
SM2 & & & & & \\
& 1.55 & 1.25013 & 768 & 127.42 & $8.14(16)$ & $44.94(19)$ \\
& 2.00 & 1.27702 & 432 & 135 & $8.89(17)$ & $44.91(20)$ \\
& 3.00 & 1.27352 & 432 & 147.97 & $9.76(16)$ & $45.88(19)$ \\
\hline \hline
\end{tabular}

clination $\beta$ for the SM2 crystals. This supports our earlier observation that the angle of inclination is a soft degree of freedom. ${ }^{12}$

For decreasing density, the free energy difference between stretched fcc and SM2 decreases and the lines intersect at $\varrho \simeq 1.17$, which is very close to the solid-nematic phase transition $(\varrho=1.163$ according to Ref. 1). Our data therefore confirm that SM2 is more stable than fcc at $a / b$ $=3$ and above $\varrho \simeq 1.17$.

In Table III, we compare the free energies of SM2, $\mathrm{SM} 2^{(\mathrm{cp})}$, and fcc as a function of aspect ratio, viz., for $a / b$ $=1.55,2$, and 3 . As our input configurations were produced at a fixed pressure $\left(P=46 k_{B} T / 8 a b^{2}\right)$, systems of different aspect ratios and/or structure had different densities. In order to compare them, we calculated the Gibbs free energy per particle $G / N$ by the Legendre transform of $F / N$ with respect to the volume. Figure 6 shows the Gibbs free energy per particle. (The lines are a guide to the eye only.) Again there is no difference (within the error bars) between the $\mathrm{SM} 2^{(\mathrm{cp})}$ structure and the other values of $\beta$. The superior stability of SM2 is confirmed for $a / b \geq 2$. At $a / b=1.55$, however, we find that fcc is more stable, indicating a phase transition between $a / b=1.55$ and $a / b=2.0$. (This happens to be near $a / b=\sqrt{3}$, the lower boundary of aspect ratios for which prolate ellipsoids can form crystals with maximal packing fraction $\eta=0.770732 ;{ }^{11}$ but smaller aspect ratios near this value still produce higher-than-fcc densities, so that we do not suspect a connection.)

In Fig. 7 we show an updated phase diagram of hard ellipsoids of revolution. It includes part of the results of

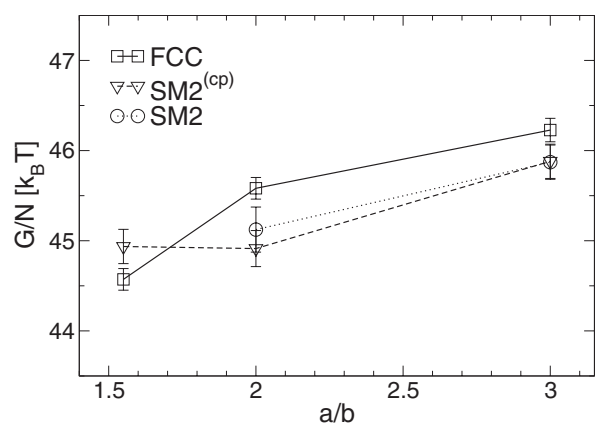

FIG. 6. Hard ellipsoids: Gibbs free energy per particle vs aspect ratio $a / b$ at $P=46 k_{B} T / 8 a b^{2}$. Lines to guide the eye. At $a / b \geq 2$, SM2 is more stable, while fec is more stable at $a / b=1.55$, implying a solid-solid phase transition in between. 


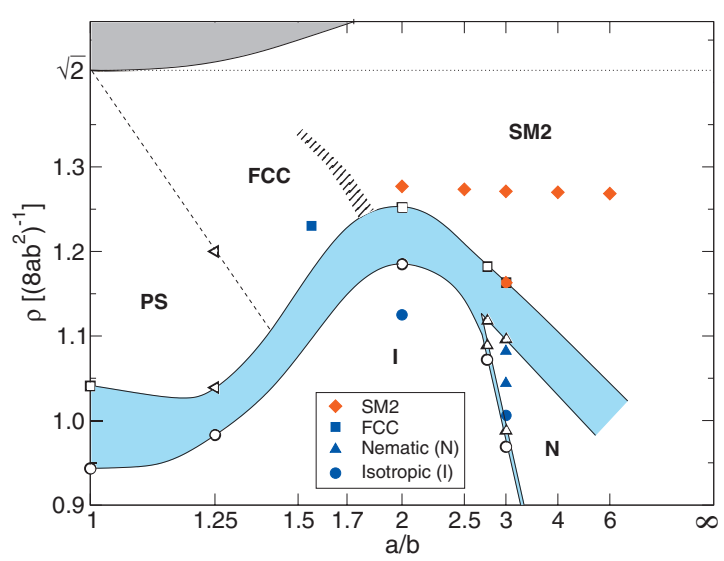

FIG. 7. Updated phase diagram of hard ellipsoids of revolution. It includes part of the results of Frenkel and Mulder (Ref. 1) (open symbols) and their suggested phase boundaries and coexistence regions. PS stands for plastic solid. The data points at $a / b=1$ are taken from Ref. 20. We have inserted our state points (this work and Ref. 7; filled symbols) and extended its high-density boundary to the maximum densities found by Donev et al. (Ref. 11), hence, including all densities possible in SM2 (recall that SM2 at maximum packing coincides with the packings of Donev et al.). In hashes we indicate a possible location of the coexistence region between fcc and SM2 (see text for details).

Frenkel and Mulder ${ }^{1}$ and their suggested phase boundaries and coexistence regions. We have inserted our state points (this work and Ref. 7) and extended its high-density boundary to the maximum densities found by Donev et al. ${ }^{11}$ hence including all densities possible in SM2 (recall that SM2 at maximum packing coincides with the packings of Donev et $a l$.). As stated above, our data imply a phase transition between SM2 and fcc near $a / b=\sqrt{3}$.

In hashes we indicate a possible location of the coexistence region according to the following argument: For spheres $(a / b=1)$ and maximum packing $(\varrho=\sqrt{2})$ the density differences among plastic solid, fcc, and SM2 vanish, so the coexistence regions among these phases should join and vanish in width either at this point, or before this point is reached. (We cannot make statements yet about the details of the approach to the sphere limit, therefore we have not drawn anything.) For $a / b>1$, and above $\varrho=\sqrt{2}$ (the dotted line), only SM2 exists, so the SM2-fcc coexistence region must lie below $\varrho=\sqrt{2}$. The packing efficiency of SM2 and the resulting entropic advantage should favor SM2 even below $\varrho$ $=\sqrt{2}$, and the stronger this advantage, the lower the transition density - hence, the downward slope of the coexistence region with increasing $a / b$. The packing advantage also dictates the increase in width of the region, since SM2 is accordingly higher in density at a given pressure. Finally, the coexistence region should pass between our state points of fcc at $a / b=1.55$ and SM2 at $a / b=2.00$. We estimate the width there from the density difference at $a / b=1.55$ and $a / b=2.00$ at pressure $P=46 k_{B} T / 8 a b^{2}$ (Table III).

\section{CONCLUSION}

In summary, we have studied crystalline phases of hard ellipsoids considering their relative stability. We calculated the absolute free energies as functions of the particle density $Q$ and the aspect ratio $a / b$ by use of a TI technique with an

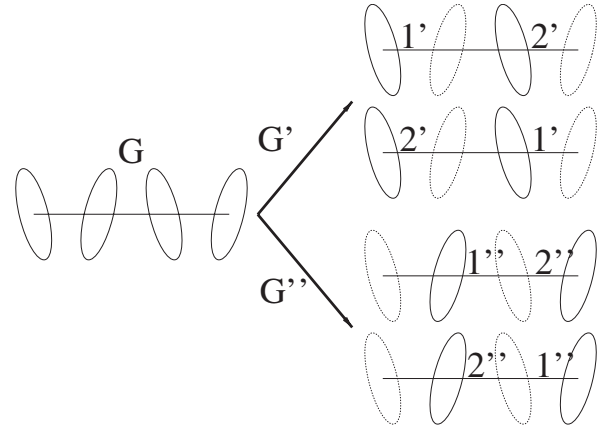

FIG. 8. Combinatorics for an EM of the SM2 type.

EM as the reference state. The integration path was sampled by an expanded ensemble method, in which the weights were adjusted by the Wang-Landau algorithm. After checking our simulations for reliability considering the test case of hard spheres, we applied our methods to ellipsoids. At pressure $P=46 k_{B} T / 8 a b^{2}$ we found no difference in the free energies of SM2 crystals with different angles of inclination $\beta$. Furthermore our results show that the SM2 phase is more stable than the stretched-fcc phase for densities $\varrho \gtrsim 1.17$ (at $a / b$ $=3$ ) and for aspect ratios $a / b \geq 2.0\left(\right.$ at $\left.P=46 k_{B} T / 8 a b^{2}\right)$. Hard ellipsoids exhibit a fcc-SM2 phase transition between $a / b$ $=1.55$ and $a / b=2.0$.

\section{ACKNOWLEDGMENTS}

We thank D. Frenkel, M. P. Allen, A. Donev, and W. A. Siebel for helpful discussions. We are grateful to the DFG (Tr6/D5 and Emmy Noether Program) for financial support and to the NIC Jülich for CPU time on the JUMP.

\section{APPENDIX: CALCULATION OF FSM2}

First, without loss of generality, we label particle $i=1$ as the carrier of the lattice. Then we write down the partition function of the SM2-EM using Eq. (4),

$$
\begin{aligned}
\mathcal{Z}^{\mathrm{SM} 2}= & \Gamma^{(N)} \int \mathrm{d} \boldsymbol{r}_{1} \int \mathrm{d} \Omega_{1} \\
& \times \int \mathrm{d} \boldsymbol{r}_{2} \cdots \mathrm{d} \boldsymbol{r}_{N} \exp \left[-\lambda_{\max } \sum_{i=2}^{N}\left(\boldsymbol{r}_{i}-\boldsymbol{r}_{0, i}\right)^{2}\right] \\
& \times \int \mathrm{d} \Omega_{2} \cdots \mathrm{d} \Omega_{N} \exp \left[-\lambda_{\max } \sum_{i=2}^{N} \sin ^{2} \theta_{i}\right] .
\end{aligned}
$$

The two trivial integrations are due to our freedom of choosing $\boldsymbol{r}_{1}$ as origin of the coordinate system and $\theta_{1}$ as some orientation in space.

$\Gamma^{(N)}$ is a combinatorial factor: We consider a lattice $G$ that consists of $N$ particles with two different orientations (distinguished by the primes in Fig. 8). We now divide $G$ into two sublattices $G^{\prime}$ and $G^{\prime \prime}$ with respect to the particle types. On these sublattices there are $(N / 2)$ ! possibilities, respectively, to position the particles on their sites. To account for the presence of the carrier on one of the sublattices the associated factorial is $(N / 2-1)$ !. Hence 


$$
\Gamma^{(N)}=\frac{\left(\frac{N}{2}\right) !\left(\frac{N}{2}-1\right) !}{\left(\frac{N}{2}\right) !\left(\frac{N}{2}\right) !}=\frac{2}{N} .
$$

The integral over the spatial coordinates can directly be carried out and leads to $\left(\pi / \lambda_{\max }\right)^{(3(N-1) / 2)}$. Hence, Eq. (A1) can be simplified to

$$
\begin{aligned}
\mathcal{Z}^{\mathrm{SM} 2} \propto & \int \mathrm{d} \theta_{2}^{\prime} \cdots \mathrm{d} \theta_{n_{1}}^{\prime} \theta_{i}^{\prime} \\
& \times \exp \left[-\lambda_{\max } \sum_{i=2}^{n_{1}} \theta_{i}^{\prime 2}\right] \int \mathrm{d} \theta_{n_{1}}^{\prime \prime} \cdots \mathrm{d} \theta_{N}^{\prime \prime} \theta_{j}^{\prime \prime} \\
& \times \exp \left[-\lambda_{\max } \sum_{j=n_{1}}^{N} \theta_{j}^{\prime 2}\right] .
\end{aligned}
$$

Here, because of the azimuthal symmetry of the problem, we already carried out the integration over $\phi$ and used the approximation $\sin \theta \approx \theta$, which was motivated before. The two remaining integrals now are related with sublattices $G^{\prime}$ and $G^{\prime \prime}$. Solving them, we find for the resulting partition function

$$
\mathcal{Z}^{\mathrm{SM} 2}=\frac{8 \pi V}{N}\left(\frac{\pi}{\lambda_{\max }}\right)^{3(N-1) / 2}\left(\frac{2 \pi}{\lambda_{\max }}\right)^{N-1}
$$

${ }^{1}$ D. Frenkel and B. Mulder, Mol. Phys. 55, 1171 (1985).

${ }^{2}$ G. J. Zarragoicoechea, D. Levesque, and J. J. Weis, Mol. Phys. 75, 989 (1992).

${ }^{3}$ M. P. Allen and C. P. Mason, Mol. Phys. 86, 467 (1995).

${ }^{4}$ P. J. Camp, C. P. Mason, M. P. Allen, A. A. Khare, and D. A. Kofke, J. Chem. Phys. 105, 2837 (1996).

${ }^{5}$ M. Letz, R. Schilling, and A. Latz, Phys. Rev. E 62, 5173 (2000).

${ }^{6}$ C. De Michele, R. Schilling, and F. Sciortino, Phys. Rev. Lett. 98, 265702 (2007).

${ }^{7}$ P. Pfleiderer, K. Milinkovic, and T. Schilling, Europhys. Lett. 84, 16003 (2008).

${ }^{8}$ A. Donev, I. Cisse, D. Sachs, E. A. Variano, F. H. Stillinger, R. Connelly, S. Torquato, and P. M. Chaikin, Science 303, 990 (2004).

${ }^{9}$ A. W. S. Sacanna, L. Rossi, and A. P. Philipse, J. Phys.: Condens. Matter 19, 376108 (2007).

${ }^{10}$ A. Bezrukov and D. Stoyan, Part. Part. Syst. Charact. 23, 388 (2006).

${ }^{11}$ A. Donev, F. Stillinger, P. Chaikin, and S. Torquato, Phys. Rev. Lett. 92, 255506 (2004).

${ }^{12}$ P. Pfleiderer and T. Schilling, Phys. Rev. E 75, 020402 (2007).

${ }^{13}$ D. Frenkel and B. Mulder, Mol. Phys. 100, 201 (2002).

${ }^{14}$ F. Wang and D. Landau, Phys. Rev. E 64, 056101 (2001).

${ }^{15}$ D. Frenkel and A. Ladd, J. Chem. Phys. 81, 3188 (1984).

${ }^{16}$ D. Frenkel and B. Smit, Understanding Molecular Simulation (Academic, New York, 2002).

${ }^{17}$ C. Vega and E. Noya, J. Chem. Phys. 127, 154113 (2007).

${ }^{18}$ J. Polson, E. Trizac, S. Pronk, and D. Frenkel, J. Chem. Phys. 112, 5339 (2000).

${ }^{19}$ C. Vega, E. Sanz, J. Abascal, and E. Noya, J. Phys.: Condens. Matter 20, 153101 (2008).

${ }^{20}$ W. Hoover and F. Ree, J. Chem. Phys. 49, 3609 (1968). 